\title{
Costa et al.: The First National Anti-TB Drug Resistance Survey in Timor-Leste
}

\section{Constantino Lopes}

National TB Programme, Ministry of Health, Timor-Leste

Debashish Kundu ( $\nabla$ debashishkundu@yahoo.com )

WHO: Organisation mondiale de la Sante https://orcid.org/0000-0002-3756-8623

\section{Ismael Da Costa Barreto}

National Health Laboratory, Ministry of Health

\section{Bernardino da Cruz}

National AIDS Programme, Ministry of Health

\section{S Siva Kumar}

National Institute for Research in Tuberculosis (WHO SNRL), Chennai

\section{Sureshbabu Ramalingam}

National TB Reference Laboratory, Dili

Anna S. Dean

Global TB Programme, World Health Organization, Geneva

\section{Vineet Bhatia}

World Health Organization Regional Office for South-East Asia

\section{Prabhu Seenivasan}

National Institute of Research in TB (WHO SNRL), Chennai

\section{Padmapriyadarsini}

National Institute of Research in TB (WHO SNRL), Chennai

\section{Olga Tosas Auguet}

Global TB Programme, World Health Organization, Geneva

\section{Research}

Keywords: Anti-TB Drug Resistance Survey (DRS), specimen collection and transportation (SC\&T), molecular diagnosis, Timor-Leste, Covid-19, survey, National TB Programme (NTP), research, rifampicin resistance, multi-drug resistant tuberculosis (MDR-TB), World Health Organization ( WHO)

Posted Date: June 7th, 2021

DOI: https://doi.org/10.21203/rs.3.rs-552154/v1 
License: (c) (i) This work is licensed under a Creative Commons Attribution 4.0 International License. Read Full License 


\section{Abstract}

\section{Background}

In the absence of reliable data, a national drug resistance survey (DRS) was implemented for the first time in Timor-Leste (TL) in 2019 to assess the prevalence of drug resistance among new and previously treated pulmonary TB patients in the country to guide resource allocation and to strengthen existing systems for the diagnosis, treatment and care of rifampicin-resistant (RR) TB.

\section{Methods}

This nation-wide cross-sectional survey was conducted from January 2019 until September 2019 targeting all new and previously treated sputum smear-positive pulmonary TB patients in the country. Sputum samples from enrolled cases were submitted to the National TB Reference Laboratory for molecular confirmation of pulmonary TB and to determine resistance to rifampicin by Xpert MTB/RIF. Culture was performed in parallel on solid media, and culture isolates from a sub-sample of confirmed TB cases were shipped to the WHO Supranational TB Reference Laboratory in Chennai, India for whole genome sequencing for quality assurance purposes. An electronic web-based system was used for entry and storage of the data from the DRS. Survey summary statistics, data cross-tabulations and analysis of potential risk factors of rifampicin-resistant TB (RR-TB) were conducted using R statistical software (version 3.5.2).

Results

A total of 953 sputum-smear positive patients were enrolled into the study, of which 917 were confirmed as positive for TB by either Xpert MTB/RIF or culture. Rifampicin resistance was detected among $0.6 \%$ (95\% $\mathrm{Cl} 0.2-1.3)$ of new cases and $2.7 \%(95 \% \mathrm{Cl} 0.5-8.2)$ of cases previously treated with anti-TB drugs, using Xpert MTB/RIF.

\section{Conclusion}

The prevalence of RR-TB in Timor-Leste is relatively low compared to the estimated prevalence of RR-TB in the WHO South-East Asia Region $(2.5 \%$ [95\% Cl 1.9-3.3] among new cases and $14 \%$ [95\% $\mathrm{Cl} 7.7-21]$ among previously treated cases). This might be attributed to the late introduction of rifampicin into the maintenance phase of first-line treatment for TB in Timor-Leste and the good treatment success rate among TB patients (88\% in new and relapse cases in 2018), although treatment coverage remains notably low.

The rapid sputum collection and transportation mechanism implemented in the survey demonstrates its feasibility in low resource settings and should be replicated for routinely transporting TB specimens from microscopy labs to GeneXpert sites, addressing barriers to accessing TB services in remote, hilly areas. Establishment of in-country capacity for rapid molecular diagnostics for both first- and second-line DST, 
such as the line probe assay, is an immediate need for achieving universal drug susceptibility testing (DST) to guide appropriate patient management.

\section{Introduction}

Timor-Leste is a low-middle income young island nation with a population of about 1.2 million and an annual population growth rate of $1.8 \%$. The country is in the eastern end of the Indonesian archipelago and is divided into 12 municipalities and one special economic zone (Oecusse). The country has an area of approximately $15,000 \mathrm{~km}^{2}$, a large part of which consists of hilly and mountainous terrain which is difficult to access. The population is thus scattered and there are large distances between people's residences and the closest TB diagnostic centre. The country has the second highest estimated tuberculosis (TB) incidence rate and highest estimated mortality rate in the South-East Asia Region ${ }^{1,2}$.

In 2018, WHO estimated a burden of 3.1\% (1.1\%-6.1\%) rifampicin-resistant (RR) TB among new cases, and $15 \%(7.8 \%-23 \%)$ among retreatment TB cases for Timor-Leste ${ }^{3}$. Accordingly, it was estimated that 240 cases of RR-TB (a rate of 19/100,000 population) ${ }^{3}$ are emerging annually in the country. These presurvey estimates were model-based, and not based on country data due to the lack of a continuous surveillance system for drug-resistant (DR) TB. The number RR-TB cases that were detected and notified between 2013-2018 as per the WHO Global TB Reports ranged from 2 to 12. In 2019, 9 were laboratory confirmed RR-TB cases. $52 \%$ of pulmonary TB cases in 2019 were bacteriologically confirmed, and only $11 \%$ (458/4050) of new and relapse cases reported using a WHO-recommended rapid diagnostic - Xpert MTB/RIF as the initial diagnostic test.

The first national DRS was conducted by the National TB Programme (NTP) and the National TB Reference Laboratory (NTRL) in 2019, with the technical assistance from the World Health Organization (WHO) and the National Institute of Research in Tuberculosis (NIRT), Chennai, India (a WHO Supranational TB Reference Laboratory, SNRL).

The primary objective of the DRS was to determine the prevalence of resistance to rifampicin among new and previously treated sputum smear-positive pulmonary TB cases in Timor-Leste, to inform the planning of the programmatic management of DR-TB (PMDT), including the roll-out of the WHO-recommended alloral shorter regimen for RR-TB ${ }^{4}$, and guide the resource needs. The secondary objectives were to describe the sociodemographic and clinical characteristics of bacteriologically-confirmed pulmonary TB patients; investigate potential risk factors for RR-TB; establish baseline data for surveillance of DR-TB in order to allow the observation of trends over time; and strengthen the routine surveillance of anti-TB drug resistance in Timor-Leste.

\section{Methods}

\section{Study population}


The national cross-sectional study targeted all newly registered (new and previously treated) pulmonary TB patients in the country who were sputum smear-positive by microscopy. Following a one-month pilot to test all study procedures, eligible patients were enrolled into the survey over nine months (from $21 \mathrm{st}$ January 2019 to 5th September 2019). Clinically diagnosed TB patients (i.e. those diagnosed without bacteriological confirmation of TB by microscopy), extra-pulmonary TB patients, sputum smear-negative cases, patients who had already received more than seven days of treatment in their current treatment course, and prisoners were excluded from enrolment.

\section{Study design and sampling strategy}

STROBE statement was followed to include check-list items in reporting this cross-sectional study ${ }^{5}$. The survey comprised $100 \%$ sampling of all Community Health Centres (CHCs) in the country offering microscopy diagnosis of TB $(n=76)$. The enrolment period was fixed across all $\mathrm{CHCs}$ to ensure that the final sample was self-weighted by the TB caseload in each facility.

The sample size calculation was based on the number of new smear positive TB cases in Timor-Leste in $2017(n=1511)$ and assumed 3.3\% expected prevalence of RR-TB among new cases of bacteriologically confirmed pulmonary TB (WHO Global TB Report 2018) ${ }^{6}$. The recommended simple random sampling formula with a finite population correction was used ${ }^{7}$ to calculate the sample required to estimate the proportion of RR-TB to an absolute precision of $1 \%$ and a $95 \%$ level of confidence. The sample was increased to account for $20 \%$ potential losses of samples due to testing errors or lost/contaminated samples, to a total of 846 new bacteriologically confirmed pulmonary TB cases. A fixed enrolment period of 8 months (calculated as [846/1511] *12 and allowing for one extra month to account for any potential unforeseen circumstances) was set across all $\mathrm{CHCs}$ to attain the required sample. All eligible previously treated cases were enrolled opportunistically at all CHCs throughout this period.

\section{Patient enrollment}

At CHCs, all eligible patients presenting consecutively to the facility for diagnosis of pulmonary $\mathrm{TB}$, were asked to produce two sputum samples (spot and morning) for smear microscopy examination following routine practice. Patients were then enrolled following informed written consent, if at least one sputum sample was positive by smear microscopy. Following the enrolment, voluntary HIV testing was conducted as per national guidelines (provider-initiated testing and counselling). Clinical, demographic and previous anti-TB treatment history data were then collected from each patient through a questionnaire (DRS Clinical Information Form [CIF]), which was completed by the TB focal person at the CHC.

\section{Sample collection and laboratory procedures}


The two sputum samples collected from the eligible patients at CHCs were transported to NTRL, Dili, maintaining a cold chain and within a target of 3-5 days. At NTRL, both samples again underwent sputum smear microscopy and inoculation on solid (Lowenstein Jensen) media. Morning samples were also tested by Xpert MTB/RIF according to manufacturer's instructions. Colonies were scraped from both solid culture slants and diluted in Phosphate Buffer Solution (PBS). One PBS aliquot was kept at NTRL for back-up storage $(-20 \circ C)$. A second PBS aliquot was shipped to Chennai supranational reference laboratory (SNRL) maintaining a cold chain, following the IATA regulations for biological substances, Category A infectious material (UN33738). For the purpose of validating Xpert MTB/RIF resistance test results, we sought to conduct whole genome sequencing (WGS) at Chennai SNRL for approximately 5$10 \%$ of TB isolates with rifampicin resistance results by Xpert MTB/RIF. For isolates that were "MTB not detected" or "MTB detected with indeterminate rifampicin resistance result" by Xpert MTB/RIF, resistance to rifampicin was evaluated by phenotypic DST (pDST) in liquid culture (BACTEC MGIT), if the culture isolate was confirmed as Mycobacterium tuberculosis at the SNRL.

WGS was conducted using Illumina HiSeq X Ten system (Illumina, San Diego, CA, USA), following extraction of genomic DNA from the clinical isolates using the CTAB (cetyl trimethylammonium bromide) method. DNA was purified using the Genomic DNA Clean and Concentrator kit and assessed for quality and quantity using Nano Drop and Qubit dsDNA Assay kits (Thermo Fisher Scientific, Waltham, MA, USA). Libraries were prepared using NEBNext Ultra DNA Library preparation kit (Illumina, San Diego, CA, USA). For the subset of isolates tested by WGS, mutations conferring resistance to isoniazid, ethambutol, streptomycin, pyrazinamide and fluoroquinolones were also examined.

\section{Data management}

An electronic web-based DRS database (https://tb.ms.gov.tl/tb1) ${ }^{9}$ was designed prior to the start of the survey for the purposes of data entry and storage of the data from the DRS. The DRS database was designed to meet the requirements of confidentiality principles and was password protected and backedup daily to a secure server.

The database contained all the data fields from patient questionnaires as well as all NTRL and SNRL laboratory test results. A separate section of the database was designed for the electronic tracking of samples, shipments, and reports of laboratory results to $\mathrm{CHCs}$.

\section{Data analysis}

Survey summary statistics, data cross-tabulations and analysis of potential risk factors of RR-TB were conducted using R statistical software (version 3.5.2) ${ }^{10}$. For each anti-TB drug, the prevalence of resistance along with the corresponding $95 \%$ confidence intervals $(95 \% \mathrm{Cl})$, was estimated by fitting a logistic regression and then taking the inverse logit of the model coefficient and confidence interval. Resistance to rifampicin was determined by considering both Xpert MTB/RIF and WGS test results, with 
the latter providing a conclusive result in case of discrepancies. Associations between RR-TB and clinical and demographic traits were explored in univariate logistic regression analyses adjusted by previous antiTB treatment history. Measures of association for potential predictors were summarised by odds ratios with the corresponding $95 \%$ confidence intervals.

\section{ETHICAL CLEARANCE}

Ethical clearance was obtained from the Instituto Nacional da Saude (INS), Ministry of Health (MOH).

\section{Results}

\section{Clinical and demographic characteristics of the TB population}

A total of 953 eligible patients were enrolled from 69s CHC across 12 municipalities and one special economic zone over the study period. Seven $\mathrm{CHCs}$ did not report any TB patients. The median time taken from sample collection to receipt of the samples at NRTL was 2 days (interquartile range of 1-3 days). Approximately $19.3 \%$ (184/953) of the samples took more than 4 days to arrive to NTRL.

Out of 953 enrolled patients, 917 were confirmed as pulmonary TB cases by either Xpert MTB/RIF and/or culture (Figure 1). More than half of bacteriologically confirmed TB patients were male (57.1\%), and 8.0\% had been previously treated for TB (Table 1 ). Only $2.1 \%$ of TB cases were children aged $<15$ years. More TB cases were identified from individuals aged 15-34 years (45.9\%). A total of 12 (1.3\%) patients were coinfected with HIV. Most cases were detected from the capital, Dili, and the Ermera municipality, which together comprised $59.4 \%$ of the cases (Table 1 ). The age and sex population structure for bacteriologically confirmed pulmonary TB patients enrolled in the study $(n=917)$ is shown in Figure 2.

\section{Sample processing workflow at NTRL and SNRL:}

Out of 953 patients enrolled into the study based on smear microscopy findings at CHCs, 913 were confirmed as pulmonary TB patients by Xpert MTB/RIF and a further four were confirmed by culture $(n=917)$. See Figure 1. Out of those confirmed as pulmonary TB by Xpert MTB/RIF, seven were RR-TB and 905 were RS-TB. Rifampicin resistance was indeterminate by Xpert MTB/RIF for one case. Phenotypic DST for this indeterminate specimen, plus four isolates that were confirmed as MTB by culture, showed all five isolates were RS-TB, totaling 7 RR-TB isolates plus 910 RS-TB isolates by Xpert MTB/RIF and/or culture.

WGS was conducted for validation purposes on 65 isolates (29\% of RR-TB (2/7) and $7 \%$ of RS-TB (63/910) by Xpert MTB/RIF or PDST). Of these, one isolate was excluded due to inconclusive speciation results, resulting in non-interpretable resistance profiling (Figure 1). The original test results agreed with 
the WGS validation results for $62 / 64$ isolates (97\%). WGS re-classified one RR-TB by Xpert MTB/RIF as susceptible to rifampicin, and one RS-TB by Xpert MTB/RIF as resistant to rifampicin. For the case of the isolate that was confirmed as resistant by WGS, a rare mutation associated with rifampicin resistance (Ser441GIn) was identified in the RRDR region. This was an unusual double mutation -where two consecutive nucleotides are replaced by two different nucleotides on the genome- leading to rpoB $441^{\text {st }}$ codon TCG being replaced by CAG (HGVS nomenclature on rpoB gene sequence: c.1321_1322delinsCA). No resistance mutations were identified in the case of the isolate that was confirmed as susceptible by WGS.

\section{Resistance to anti-TB drugs}

The prevalence of rifampicin resistance among new cases was $0.6 \%(95 \% \mathrm{Cl} 0.2-1.3)$ and that among previously treated TB cases was $2.7 \%(95 \% \mathrm{Cl} 0.5$ - 8.2). The prevalence of rifampicin resistance among these groups combined was $0.8 \%(95 \% \mathrm{Cl} 0.3-1.5)$. See Table 2 . Of the two RR-TB with WGS test results, one also had resistance mutations for isoniazid, ethambutol and streptomycin, and a second one showed no resistance mutations to evaluated drugs (i.e. isoniazid, ethambutol, streptomycin, pyrazinamide and fluoroquinolones). Among the 64 isolates with WGS test results, no mutations conferring resistance to pyrazinamide or fluoroquinolones were identified; mutations conferring resistance to isoniazid, ethambutol and streptomycin were identified from seven, two and one isolates respectively. Due to the low testing coverage of $M$. tuberculosis by WGS (7\% [64/917]), resistance estimates and corresponding $95 \%$ confidence intervals for drugs other than rifampicin were not calculated in this study.

No factors were associated with RR-TB in univariate logistic regression analyses (Table 3). Multiple regression analysis was not conducted due to the small number of RR-TB cases $(n=7)$ identified.

\section{Discussion}

This is the first national survey of drug resistance among people with TB in Timor-Leste; most TB cases identified were male and under 55 years of age. Notably, children $<15$ years old account for $11 \%$ of TB globally, but only represented $2 \%$ in Timor-Leste during this 9-month period. The WHO Global TB Report $2020^{11}$ estimated that $8 \%$ of notified TB cases were children in Timor-Leste, including both clinically diagnosed and bacteriologically confirmed pulmonary and extra-pulmonary TB. This difference may be partially explained by the DRS enrolling only the bacteriologically confirmed pulmonary TB cases. Obtaining bacteriological confirmation of TB in children remains a challenge.

The overall prevalence of RR-TB was $0.8 \%$ (95\% $\mathrm{Cl} 0.3-1.5 \%)$, with $0.6 \%$ (95\% $\mathrm{Cl} 0.2-1.3 \%$ ) among new TB patients and $2.7 \%(95 \% \mathrm{Cl} 0.5-8.2 \%)$ among previously treated TB patients. This is notably lower than previous modelled estimates prior to the survey. The results of this survey show a lower prevalence than in neighbouring Indonesia (2.4\% (1.8-3.3) among new cases and 13\% (9-18) among previously treated cases) and in the WHO Southeast Asia Region (2.5\% (1.9-3.3) among new cases and 14\% (7.7-21) among 
previously treated cases) ${ }^{12}$. The lower levels of rifampicin resistance in Timor-Leste may relate to the fact that rifampicin was only introduced into the four-month continuation phase of the first-line treatment regimen from the second quarter of 2015 (in line with the WHO-recommended regimen of 2HRZE+4RH), which was much later than in neighbouring countries. The lower level of rifampicin resistance found in this study in Timor-Leste might also be attributed to a good treatment success rate among TB patients who were enrolled on treatment ( $88 \%$ in new and relapse TB cases in 2018). However, treatment coverage is low (63\%) compared to the estimated number of incident TB cases in Timor-Leste, and this also may have led to a lower selection pressure on DR-TB strains. Transmission rates of TB remain high, with the second highest incidence rate (498 cases per 100,000 population) in the WHO South-East Asia Region and among the top ten in the world.

This survey did not find any significant risk factors associated with resistance to rifampicin, likely as a result of the small number of RR-TB cases identified (7 cases only).

Although this study did not formally evaluate resistance to drugs other than rifampicin due to the limited number of sequenced isolates, mutations conferring resistance to isoniazid, ethambutol and streptomycin were identified, with 7/64 isolates displaying genotypic resistance to isoniazid. No resistance-conferring mutations for pyrazinamide or fluoroquinolones were observed among sequenced samples, which may bode well for the management of DR-TB in the country. However, WGS was not systematically performed for all samples.

The WHO End TB Strategy calls for the early diagnosis of TB and universal drug susceptibility testing (DST), highlighting the critical role of laboratories for rapidly and accurately detecting TB and drug resistance. DR-TB is a public health crisis and a major threat to global TB control. WHO recommends the use of Line Probe Assay (LPA) as a rapid diagnostic test for the detection of rifampicin, isoniazid, fluoroquinolone and pyrazinamide resistance ${ }^{12}$. A Regional Green Light Committee (r-GLC) mission in 2018 in Timor-Leste recommended investment by the country in LPA ${ }^{\mathbf{1 3}}$, to rapidly detect isoniazid and fluroquinolone resistance to inform appropriate patient management. The TB Joint Monitoring Mission (JMM) conducted in Timor-Leste in 2019 also recommended that the NTP consider introducing LPA capacity into the NTRL. By mid-2021, it is anticipated that all bacteriologically-confirmed TB patients will be offered DST for rifampicin and isoniazid, as well as fluoroquinolones among cases of RR-TB, thus facilitating timely clinical management of TB patients and appropriate implementation of WHO guidelines on use of all-oral shorter regimen.

This study showed good agreement between Xpert MTB/RIF and WGS results (97\%), suggesting that nationwide diagnosis of RR-TB through Xpert MTB/RIF is adequate in this setting. However, WGS also identified an unusual double mutation in the RRDR region of the genome which was missed by Xpert MTB/RIF. The occurrence of mutations missed by routine diagnostic tools should be regularly monitored in the region through collaborations with supranational referral laboratories, SNRLs, for quality assurance purposes. One RR-TB case by Xpert MTB/RIF, later found to be susceptible to rifampicin by WGS, may be explained by the analysis of an heteroresistant sample by Xpert MTB/RIF and/or the preferential 
selection of a susceptible strain upon culturing a sputum sample harbouring a mixed infection, prior to WGS. We also found that of the 40 samples that tested negative to M. tuberculosis by Xpert MTB/RIF at NTRL, 4 were positive by culture. It is possible that the bacterial load may have been too low for detection by Xpert MTB/RIF. Similar findings have been reported in other studies ${ }^{14}$.

Currently, eight GeneXpert machines are functional in Timor-Leste. An additional two machines of 16 modules have been received for the Covid-19 response, which can also be used by the NTP for TB diagnosis. Linkages with the GeneXpert machines will also be prioritised for intensified case finding at all facilities with a high outpatient caseload ${ }^{15}$. During the survey, sputum samples were transported from all the municipalities in the country to the GeneXpert sites and NTRL with minimal delay (median of 2 days) through human carriers who were adequately incentivized for this work. Delay was observed in sample shipment from NTRL, Dili, to the SNRL, Chennai, and the availability of the courier agency to ship biohazard samples, which led to decreased viability and contamination of transported samples. Any discordance between Xpert and LPA tests could be resolved by phenotypic testing, once capacity is established in-country, and appropriate clinical evaluation of the patient

For routine patient management and surveillance of drug resistance, it is planned that sputum specimen samples will be routinely transported from all the municipalities to the eight GeneXpert sites, as per the revised NTP guidelines. An efficient specimen referral system is expected to be developed in the next Global Fund cycle with the support of partners, including linking with active TB case finding initiatives led by the NTP and community volunteers The same mechanism could be replicated for routinely transporting samples from the microscopy labs to the GeneXpert sites and could address barriers to accessing TB diagnosis and care from remote, hilly terrains in this island nation.

In conclusion, the survey highlighted the need for strengthening sputum specimen transportation, establishing electronic recording and reporting, achieving NTRL accreditation, and ultimately implementing universal DST in Timor-Leste for both first- and second-line drugs. The relatively low prevalence of RR-TB in Timor-Leste is an encouraging finding, but gaps remain in obtaining bacteriological confirmation of TB and routine rifampicin testing among bacteriologically confirmed cases. This study showed that the highest burden of TB was in economically productive age groups and predominantly in male gender, as well as reproductive age groups, as in other countries. Thus, the survey highlights the significant negative impact of TB on the country's economic growth, and therefore addressing TB and DR-TB in particular, merits sustained investment.

\section{Declarations}

Source of Support: The Global Fund grant

Conflict of Interest: None declared.

Contributorship: DK developed the concept, designed and performed the experiment, analysed the data, wrote the paper, developed, edited and revised the final manuscripts; CL performed the experiments, 
reviewed and revised the paper; SB reviewed the paper; IB reviewed and revised the paper; VB reviewed and revised the paper; $\mathrm{BC}$ contributed materials; SK, PC and PP reviewed and analysed the data; OT designed the experiment, analysed the data, edited the manuscript and reviewed the paper; AD designed the experiment, analysed the data, reviewed, edited and revised the final manuscript. All authors read and approved the final manuscript.

Acknowledgments: The authors acknowledge the support provided by the staff of the National TB Programme, and National TB Reference Laboratory, Timor-Leste; National Institute for Research in Tuberculosis, Chennai (India); and technical assistance from the World Health Organization (WHO). Financial support for the drug resistance survey was provided by the Global Fund country grant.

\section{References}

1. WHO. Timor-Leste. Country Profiles. Data are as reported to WHO. Estimates of TB and MDR-TB burden are produced by WHO in consultation with countries. Available at http://www.who.int/tb/country/data/profiles/en/. Accessed on 20 September 2020

2. WHO SEARO. Annual Report 2017. Bending the curve - Ending TB. Available at: http://apps.who.int/iris/bitstream/10665/254762/1/978929022584-eng.pdf. Accessed on 15.11.2020

3. WHO Global TB Report 2019, Timor-Leste Country Profile. Available at: https://worldhealthorg.shinyapps.io/tb_profiles/?_inputs_\&lan=\%22EN\%22\&iso2=\%22TL\%22.

4. WHO consolidated guidelines on tuberculosis. Module 4: treatment - drug-resistant tuberculosis treatment. Geneva: World Health Organization; 2020. Available at https://www.who.int/publications/i/item/9789240007048

5. Vandenbroucke JP, von Elm E, Altman DG, Gøtzsche PC, Mulrow CD, et al. (2007) Strengthening the Reporting of Observational Studies in Epidemiology (STROBE): Explanation and Elaboration. PLoS Med 4: e297. Available at: www.strobe-statement.org. Accessed on 23.11.2020

6. WHO Global TB Report 2018, Timor-Leste Country Profile. Available at: https://apps.who.int/iris/handle/10665/274453

7. World Health Organization. (2015). Guidelines for surveillance of drug resistance in tuberculosis 5th Edition. Geneva: WHO.

8. UN3373 Medical Packaging. Available at: http://www.un3373.com/category-biologicalsubstances/category-b/. Accessed on 29.11.2020

9. An electronic web-based DRS database. Avaialble at: https://tb.ms.gov.tl/tb1. Accessed on 23.11.2020

10. R: A Language and Environment for Statistical Computing. R Foundation for Statistical Computing, Vienna, Austria

11. WHO Global TB Report 2020. Available at: https://www.who.int/teams/global-tuberculosisprogramme/tb-reports. Accessed on 23.11.2020 
12. Update on the use of nucleic acid amplification tests to detect TB and drug-resistant TB: rapid communication (who.int). January 2021.

13. Report of the Regional Green Light Committee (r-GLC) mission in 2018 in Timor-Leste. WHO

14. Agrawal M, Bajaj A, Bhatia V, Dutt S. Comparative Study of GeneXpert with ZN Stain and Culture in Samples of Suspected Pulmonary Tuberculosis. J Clin Diagn Res. 2016;10(5):DC09-DC12. doi:10.7860/JCDR/2016/18837.77558.

15. Comprehensive TB guideilnes for the national TB programme, Timor-Leste, V Edition. 2020. Available at: https://apps.who.int/iris/handle/10665/333647. Accessed on 23.11.2020

\section{Tables}

Table 1: Demographic and clinical characteristics of bacteriologically confirmed TB patients enrolled in the survey.

\begin{tabular}{|c|c|c|c|c|c|c|}
\hline \multirow[b]{3}{*}{ Patient Characteristics } & \multirow{2}{*}{\multicolumn{2}{|c|}{$\begin{array}{c}\text { New } \\
n=844\end{array}$}} & \multirow{2}{*}{\multicolumn{2}{|c|}{$\begin{array}{c}\text { Previously Treated } \\
n=73\end{array}$}} & \multirow{2}{*}{\multicolumn{2}{|c|}{$\begin{array}{c}\text { Combined } \\
n=917\end{array}$}} \\
\hline & & & & & & \\
\hline & Number & $\%$ & Number & $\%$ & Number & $\%$ \\
\hline \multicolumn{7}{|l|}{ Gender } \\
\hline Male & 479 & 56.8 & 45 & 61.6 & 524 & 57.1 \\
\hline Female & 365 & 43.2 & 28 & 38.4 & 393 & 42.9 \\
\hline \multicolumn{7}{|l|}{ Age } \\
\hline $0-14$ & 19 & 2.3 & 0 & 0.0 & 19 & 2.1 \\
\hline $15-24$ & 212 & 25.1 & 11 & 15.1 & 223 & 24.3 \\
\hline $25-34$ & 179 & 21.2 & 19 & 26.0 & 198 & 21.6 \\
\hline $35-44$ & 111 & 13.2 & 7 & 9.6 & 118 & 12.9 \\
\hline $45-54$ & 107 & 12.7 & 18 & 24.7 & 125 & 13.6 \\
\hline $55-64$ & 107 & 12.7 & 6 & 8.2 & 113 & 12.3 \\
\hline $65+$ & 109 & 12.9 & 12 & 16.4 & 121 & 13.2 \\
\hline
\end{tabular}

HIV Status ${ }^{1}$

$\begin{array}{rcccccc}\text { Negative } & 832 & 98.7 & 72 & 98.6 & 904 & 98.7 \\ \text { Positive } & 11 & 1.3 & 1 & 1.4 & 12 & 1.3\end{array}$

Municipality

\begin{tabular}{rcccccc} 
Aileu & 23 & 2.7 & 2 & 2.7 & 25 & 2.7 \\
Ainaro & 14 & 1.7 & 0 & 0.0 & 14 & 1.5 \\
Baucau & 43 & 5.1 & 4 & 5.5 & 47 & 5.1 \\
Bobonaro & 68 & 8.1 & 6 & 8.2 & 74 & 8.1 \\
Covalima & 37 & 4.4 & 4 & 5.5 & 41 & 4.5 \\
Dili & 297 & 35.2 & 28 & 38.4 & 325 & 35.4 \\
Ermera & 211 & 25.0 & 9 & 12.3 & 220 & 24.0 \\
Lautem & 26 & 3.1 & 5 & 6.8 & 31 & 3.4 \\
Liquica & 31 & 3.7 & 5 & 6.8 & 36 & 3.9 \\
Manatuto & 24 & 2.8 & 3 & 4.1 & 27 & 2.9 \\
Manufahi & 17 & 2.0 & 4 & 5.5 & 21 & 2.3 \\
Oecusse & 33 & 3.9 & 2 & 2.7 & 35 & 3.8 \\
Viqueque & 20 & 2.4 & 1 & 1.4 & 21 & 2.3 \\
\hline
\end{tabular}


${ }^{1}$ One new TB patient with missing HIV status

Table 2: Prevalence of rifampicin resistance among TB patients in the survey, overall and by previous anti-TB treatment history.

\begin{tabular}{|c|c|c|c|}
\hline & Total TB Cases & RR-TB & $\%(95 \% \mathrm{Cl})$ \\
\hline New Patients & 844 & 5 & $0.6(0.2-1.3)$ \\
\hline Previously Treated Patients & 73 & 2 & $2.7(0.5-8.2)$ \\
\hline All Patients & 917 & 7 & $0.8(0.3-1.5)$ \\
\hline
\end{tabular}

RR-TB: rifampicin resistant tuberculosis; 95\% Cl: 95\% confidence intervals

Table 3: Risk Factors for development of rifampicin-resistant TB

\begin{tabular}{|c|c|c|c|c|c|}
\hline Variable & Level & Total TB Cases & RR-TB Cases & Odds Ratio $(95 \% \mathrm{CI}$ ) & p-value \\
\hline Treatment History & New & 844 & 5 & & 0.11 \\
\hline & Previously Treated & 73 & 2 & $4.7(0.7-22.4)$ & \\
\hline Gender & Male & 524 & 4 & & 0.96 \\
\hline & Female & 393 & 3 & $1.0(0.2-4.8)$ & \\
\hline HIV Status 1 & Negative & 904 & 7 & & NA \\
\hline & Positive & 12 & 0 & NA & \\
\hline Age & $15-24$ & 223 & 1 & & 0.99 \\
\hline & $0-14$ & 19 & 0 & NA & \\
\hline & $25-34$ & 198 & 2 & $4.5(0.2-43.4)$ & \\
\hline & $35-44$ & 118 & 1 & $1.8(0.1-47.1)$ & \\
\hline & $45-54$ & 125 & 1 & $1.4(0.1-36.3)$ & \\
\hline & $55-64$ & 113 & 1 & $2.0(0.1-50.1)$ & \\
\hline & $65+$ & 121 & 1 & $1.6(0.1-41.4)$ & \\
\hline Municipality & Other municipalities & 592 & 2 & & 0.06 \\
\hline & Dili Capital & 325 & 5 & $4.5(1.0-31.7)$ & \\
\hline
\end{tabular}

Univariate logistic regression analyses of key demographic and clinical variables as potential predictors of rifampicin resistance. Analyses were adjusted by treatment history. Variable levels with zero cases were excluded from the analyses; consequently, no analysis was conducted for HIV, but numbers of rifampicin resistant cases disaggregated by HIV status are included in the table for reference.

${ }^{1}$ HIV status was missing for one patient. 


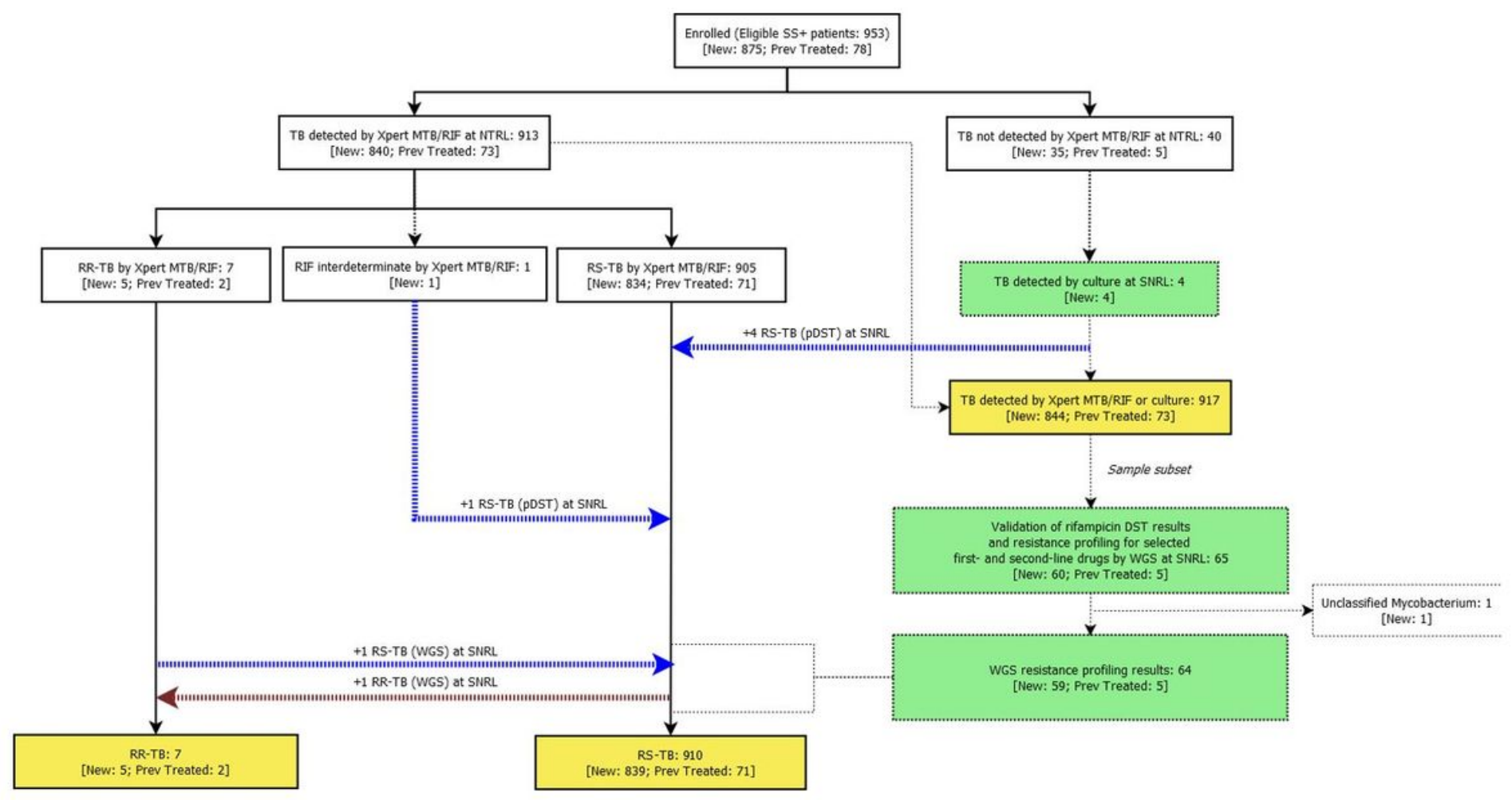

\section{Figure 1}

Timor-Leste's anti-TB drug resistance survey sample processing workflow. SS+ = sputum smear-positive (on microscopy); Prev Treated = previously treated; RIF = rifampicin; RR-TB = rifampicin-resistant TB (including MDR-TB); RS-TB = rifampicin-susceptible TB; pDST = phenotypic DST; WGS = whole genome sequencing; NTRL = National TB Reference Laboratory; SNRL = Supranational Laboratory. 

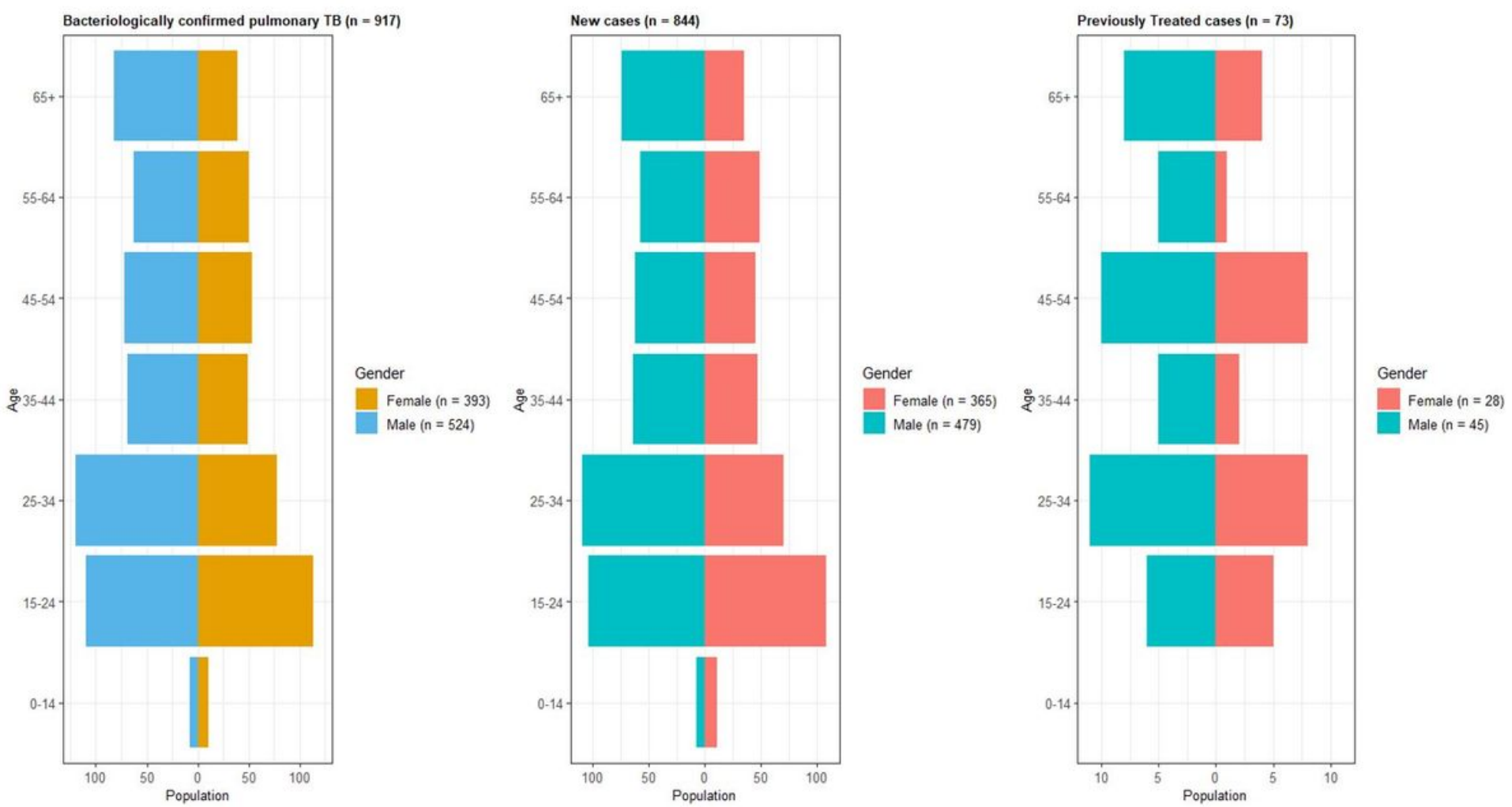

Figure 2

Age/Sex population structure of bacteriologically confirmed pulmonary TB patients 